\title{
Chest radiography and high resolution computed tomography in the evaluation of workers exposed to silica dust: relation with functional findings
}

\author{
D Talini, P L Paggiaro, F Falaschi, L Battolla, M Carrara, M Petrozzino, E Begliomini, \\ C Bartolozzi, C Giuntini
}

\begin{abstract}
Objectives-To compare the usefulness of high resolution computed tomography (HRCT) with chest radiography (CR) in the diagnosis and assessment of severity of silicosis.
\end{abstract}

Methods-27 workers exposed to silica underwent CR, HRCT, and pulmonary function tests. Two experienced readers independently evaluated CR by International Labour Office classification, and grouped the results into four categories. HRCT categories of nodule profusion and the extent of emphysema were graded on a four point scale; in 20 subjects the percentage distribution of lung densities were measured by HRCT. Results-Concordance between readers was higher for HRCT than for CR ( $K$ statistic $=0.49$ and 0.29 respectively). There was poor concordance between CR and HRCT in the early stages of silicosis. No significant difference in pulmonary function tests was found among different CR categories, but forced expiratory volume in one second $\left(F E V_{1}\right)$, maximal expiratory flow at $50 \%$ and $75 \%$ of FVC $\left(M F_{50}, M E F_{75}\right)$, and diffusion capacity significantly decreased with increasing HRCT categories. Subjects with simple silicosis detected by HRCT had a lower FEV 1 than subjects without silicosis, whereas subjects with conglomerated silicosis showed higher residual volume and functional residual capacity than subjects with simple silicosis. These relations were not affected by smoking or symptoms of chronic bronchitis. Different grades of emphysema detected by HRCT were significantly different in diffusion capacity. Only the HRCTs of the lowest and the highest categories of profusion of parenchymal opacities were significantly different in their distribution of density classes. Conclusion-HRCT is more reproducible and accurate than $C R$, as suggested by the higher agreement between readers and the better correlation with pulmonary function tests, irrespective of smoking and chronic bronchitis; however, these data do not support the hypothesis that HRCT is more sensitive than CR in the early detection of silicosis.

(Occup Environ Med 1995;52:262-267)
Keywords: silicosis; chest radiography; computed tomography

Radiographic assessment is paramount in the diagnosis of silicosis, ${ }^{1}$ particularly the standardised chest $x$ ray film classification. ${ }^{2}$ The reproducibility and the sensitivity of chest radiography (CR) to diagnose early silicosis is not very high ${ }^{34}$ and some alternative methods have been evaluated. Functional measurements have been shown to be of little use in the diagnosis of silicosis, because they can be normal even in the presence of high profusion of small opacities, and they have been used more frequently to assess the severity of functional impairment due to the associated chronic obstructive pulmonary disease (COPD). ${ }^{56}$ Markers of activity of the interstitial disorder (like broncho-alveolar lavage or gallium-67 scan) have been shown to be abnormal in the early stages of silicosis, ${ }^{78}$ but their usefulness in the diagnosis of early silicosis in individual people has not been shown.

Since 1983, several reports have shown that computed tomography (CT) and high resolution computed tomography (HRCT, thin collimation slices performed with a small field of view and targeted by means of high spatial frequency algorithm) were very informative in detecting silicotic parenchymal abnormalities and in quantifying their extent and severity, ${ }^{9}{ }^{10}$ as they have been in other diffuse interstitial lung diseases. ${ }^{11-13}$ Several studies have been carried out to compare the CT findings with the conventional radiographic patterns. Many authors showed that CT does image the parenchimal disease more clearly and identifies significantly more coalescence or large opacities than plain film..$^{914}$ On the contrary, other authors reported the relative insensitivity of lung CT in detection of mild silicosis. ${ }^{1516}$ Lung CT was useful in the evaluation of changes in emphysema that could explain the functional abnormalities found in silicosis. ${ }^{16-18}$

The aim of this study was to compare CR and HRCT in a group of subjects exposed to silica dust and with silicosis of different severity; furthermore, the relation between the radiological assessment and pulmonary function tests was evaluated, taking into account the simultaneous presence of the associated COPD. 


\section{Subjects and methods}

SUBJECTS

We studied 27 subjects ( 26 men, one woman; eight smokers, six non-smokers, 13 exsmokers) with mean (SD) age of $57(8 \cdot 8)$ (range 44-76) years. All of them had been exposed to silica dust in mines, in glass and pottery industries, and in the building industry. Most of them had been diagnosed as having silicosis, based on a history of exposure to silica and radiographic changes consistent with silicosis (presence of rounded or irregular small opacities with profusion $\geqslant 1 / 0$ on the International Labour Office (ILO) classification).

\section{CHEST RADIOGRAPHY}

Standard high kilovoltage posteroanterior and lateral CR was carried out at maximal inspiration. The posteroanterior view of each film was graded for the profusion of opacities by two experienced observers separately (one radiologist and one pulmonary specialist) according to the ILO 1980 classification. ${ }^{2}$ To group patients with similar profusions of disease, four categories were defined on the basis of the same ILO profusion grades: category $0=$ profusion grades $0 /-, 0 / 0,0 / 1$; category $1=$ profusion grades $1 / 0,1 / 1,1 / 2$; category $2=$ profusion grades $2 / 1,2 / 2,2 / 3$; category $3=$ profusion grades $3 / 2,3 / 3,3 /+$, associated or not with coalescence. In the presence of a discrepancy between the two readers (difference in the CR categories $\geqslant 1$ ), the CR films were read by the two readers together and a final evaluation was reached.

HIGH RESOLUTION COMPUTED TOMOGRAPHY High resolution computed tomography scans were obtained in the supine position by means of a third generation tomograph (GE CT/T 9800 , GE Sytec 3000) with a two to three second scanning time and a $512 \times 512$ matrix. The HRCT was performed with breath held after deep inspiration and a $1-1.5 \mathrm{~mm}$ section thickness at $10 \mathrm{~mm}$ intervals from the apex to the base of the lungs. The scans were performed with a small field of view (from 26 to $30 \mathrm{~cm}$ ) and targeted by means of a bone algorithm. All images were photographed at window levels and widths appropriate for lung parenchyma (level $=-500-600$, width $=$ 1200-1500) and for the mediastinum (level = 10-50, width $=250-350$ ).

All HRCT images were graded by the same two readers separately, according to the criteria of Bergin et al for the profusion of the parenchymal opacities. ${ }^{18}$ Briefly, they were classified in five categories as follows: category $0=$ no definite nodules; category $1=$ small number of nodules without disruption of vascular markings; category $2=$ many definite nodules, but with no confluence; category $3=$ confluence of nodules with disruption of vascular markings; category $4=$ confluence of nodules extending over two or more slices. The HRCT scans were also scored for the extent of nodules or emphysema with a visual scoring system; this four point system was based on estimation of the percentage of the overall area that showed emphysematous or nodular changes: grade $1=$ involvement of lung parenchyma $<25 \%$; grade $2=$ involvement of $25 \%-50 \%$ of the parenchyma; grade $3=$ involvement of $50 \%-75 \%$ of the parenchyma; grade $4=$ involvement of lung parenchyma $>75 \%$. Discordant results between the two readers were resolved as for CR.

Density measurements of lung parenchyma were performed in 20 patients. Densities by HRCT were expressed in HUs (Hounsfield Units). A mean value of density was computed separately for a right and left section of the lung parenchyma, taken in the top (at the aortic knob level) and in the middle (at the level of the right middle lobar bronchus) of the field. The distribution of different density classes at the above mentioned levels, were visualised by means of histograms.

\section{PULMONARY FUNCTION TESTS}

Each subject completed the following measurements: static lung volumes (vital capacity and its division by spirometry; functional residual capacity by multiple nitrogen washout technique), forced vital capacity (FVC) and derived indices (by Fleisher No 3 pneumotacograph), diffusion capacity of carbon monoxide (TLCO) using the single breath $\left(\mathrm{TLCO}_{\mathrm{SB}}\right)$, and steady state $\left(\mathrm{TLCO}_{\mathrm{SS}}\right)$ technique; arterial oxygen and carbon dioxide tension $\left(\mathrm{PaO}_{2}\right.$ and $\left.\mathrm{PaCO}_{2}\right)$. Lung volume and flows were measured by means of an HP Pulmonary Desk System 47804/A; reference values were obtained by Paoletti et al for the flow volume curve, ${ }^{19}$ and from Quanjer for static lung volumes. ${ }^{20}$ Diffusion capacity was obtained by means of a Morgan Resparameter MK4; reference values were derived from Cotes for the single breath technique ${ }^{21}$ and from Bates et al for the steady state technique. ${ }^{22}$

\section{STATISTICAL ANALYSIS}

The concordance in the CR and HRCT interpretations between two readers, and between CR and HRCT was evaluated by the Cohen's $\kappa$ statistic. Analysis of variance was used to compare mean values of pulmonary indices (in percentages of the predicted value) among different classes of radiological involvement, and multiple logistic regression analysis was used when the influence of smoking and chronic bronchitis on the relation between HRCT categories and spirometric impairment was considered. The distribution of the different classes of lung density in groups of subjects with different degrees of silicosis by HRCT was analysed with the KolmogorovSmirnov non-parametric test. A level of probability $<5 \%$ was considered as significant. ${ }^{23}$

\section{Results}

The agreement between two readers about the categories of parenchymal opacities on CR and HRCT was better for HRCT than for CT (Cohen's $\kappa$ statistic: $0.49 v 0.29$ respectively). The largest discrepancy occurred in the 0 and 1 categories. 
Figure 1 Concordance between $C R$ and HRCT categories (Cohen's $\kappa$ statistic: $0 \cdot 49$ ).

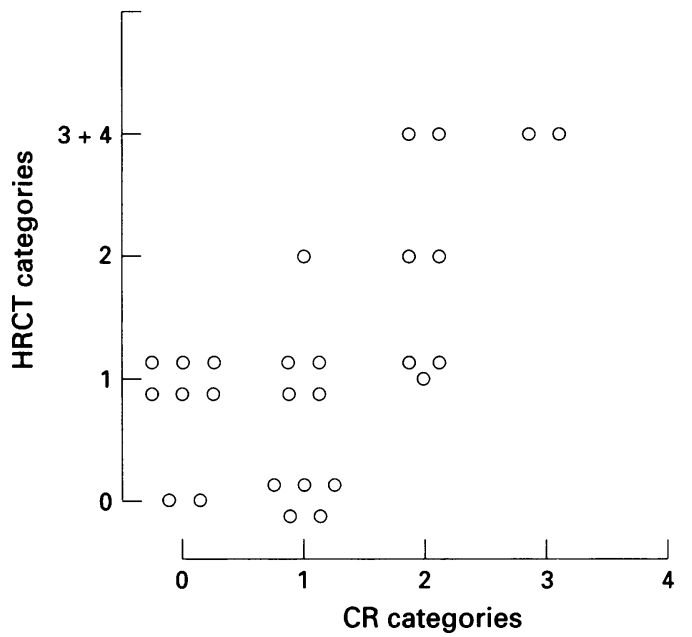

Table 1 Clinical data of 27 subjects exposed to silica dust, with different categories of profusion of small opacities by $C R$

\begin{tabular}{lllll}
\hline & 0 & 1 & 2 & 3 \\
\hline Subjects (n) & 8 & 10 & 7 & 2 \\
Mean (SD) age (y) & $57 \cdot 8(5 \cdot 9)$ & $55 \cdot 6(9 \cdot 2)$ & $57 \cdot 7(12)$ & $60(8 \cdot 5)$ \\
Smoking (n): & 2 & 5 & 1 & - \\
$\quad$ Current smokers & 4 & 4 & 5 & - \\
$\quad$ Ex-smokers & 1 & 2 & 1 & 2 \\
$\quad$ Non-smokers & $18 \cdot 7(14 \cdot 3)$ & $14 \cdot 3(13)$ & $15 \cdot 2(13 \cdot 9)$ & - \\
Mean (SD) (pack/y) & $35 \cdot 2(8)$ & $29 \cdot 2(13 \cdot 2)$ & $31 \cdot 2(12 \cdot 3)$ & $20(21 \cdot 2)$ \\
Mean (SD) silica exposure (y) & $71 \cdot 4$ & $54 \cdot 5$ & $71 \cdot 4$ & 50 \\
Chronic bronchitis (\%) & $71 \cdot 4$ & & &
\end{tabular}

Table 2 Mean (SD) values of pulmonary function tests (\% of predicted value) and blood gas concentrations in CR categories of different profusion of the parenchymal opacities (categories 2 and 3 were pooled because of low numbers of subjects)

\begin{tabular}{|c|c|c|c|}
\hline & 0 & 1 & $2+3$ \\
\hline Subjects (n) & 8 & 10 & 9 \\
\hline FVC & $96 \cdot 6(17 \cdot 6)$ & $99 \cdot 3(21 \cdot 2)$ & $96 \cdot 4(9)$ \\
\hline $\mathrm{FEV}_{1}$ & $89 \cdot 7(19 \cdot 7)$ & $94(14 \cdot 7)$ & $82 \cdot 6(9 \cdot 5)$ \\
\hline $\mathrm{MEF}_{50}$ & $73 \cdot 8(27 \cdot 4)$ & $87 \cdot 9(27 \cdot 0)$ & $57.9(18.7)^{\star}$ \\
\hline $\mathrm{MEF}_{75}$ & $57 \cdot 6(21 \cdot 2)$ & $65(29 \cdot 0)$ & $56(33 \cdot 7)$ \\
\hline $\mathrm{FEV}_{1} /$ FVC & $66 \cdot 2(9 \cdot 0)$ & $69 \cdot 9(7 \cdot 7)$ & $63.6(9)$ \\
\hline VC & $107 \cdot 8(14 \cdot 7)$ & $109(20 \cdot 0)$ & $104 \cdot 6(9)$ \\
\hline RV & $82 \cdot 3(12 \cdot 5)$ & $84(20 \cdot 8)$ & $86 \cdot 2(45 \cdot 5)$ \\
\hline FRC & $93 \cdot 7(17 \cdot 1)$ & $94.9(18 \cdot 7)$ & $103 \cdot 3(40 \cdot 4)$ \\
\hline TLC & $97.9(10.8)$ & $99 \cdot 2(15 \cdot 1)$ & $97 \cdot 5(23 \cdot 5)$ \\
\hline $\mathrm{TLCO}_{\mathrm{SB}}$ & $113.5(11.4)$ & $107 \cdot 1(43 \cdot 0)$ & $84(33 \cdot 8)$ \\
\hline TLCOss & $90 \cdot 9(26 \cdot 8)$ & $87 \cdot 8(35 \cdot 7)$ & $73 \cdot 8(24 \cdot 9)$ \\
\hline $\mathrm{PaCO}_{2}(\mathrm{~mm} \mathrm{Hg})$ & $38 \cdot 1(4 \cdot 5)$ & $39 \cdot 2(3 \cdot 6)$ & $40(2 \cdot 5)$ \\
\hline $\mathrm{PaO}_{2}(\mathrm{~mm} \mathrm{Hg})$ & $101 \cdot 0(15 \cdot 7)$ & $94 \cdot 3(12 \cdot 7)$ & $92 \cdot 5(13.6)$ \\
\hline
\end{tabular}

$\star \mathrm{P}<0.05 v$ category 1 .

Table 3 Mean (SD) values of pulmonary function tests (\% of predicted values) and blood gas concentrations in HRCT categories of profusion of parenchymal opacities (categories 2, 3, and 4 were pooled, because of low numbers of subjects)

\begin{tabular}{lccc}
\hline & 0 & 1 & $2+3+4$ \\
\hline Subjects (n) & 7 & 13 & 7 \\
FVC & $103(15 \cdot 3)$ & $92(19 \cdot 4)$ & $99 \cdot 6(12)$ \\
FEV $_{1}$ & $102 \cdot 2(11 \cdot 9)$ & $85 \cdot 1(15 \cdot 4)^{\star}$ & $81 \cdot 5(8)^{\star}$ \\
MEF $_{50}$ & $98 \cdot 6(12 \cdot 9)$ & $73 \cdot 6(28 \cdot 1)^{\star}$ & $51(1 \cdot 6)^{\star} \dagger$ \\
$\mathrm{MEF}_{75}$ & $81 \cdot 4(24 \cdot 7)$ & $57 \cdot 5(28 \cdot 4)^{\star}$ & $42 \cdot 4(17 \cdot 4)^{\star}$ \\
$\mathrm{FEV}_{1} / \mathrm{FVC}$ & $72 \cdot 4(5 \cdot 7)$ & $66 \cdot 4(10 \cdot 2)$ & $62(6 \cdot 3)^{\star}$ \\
VC & $113 \cdot 7(13 \cdot 1)$ & $102 \cdot 5(19 \cdot 1)$ & $107 \cdot 2(9 \cdot 7)$ \\
RV & $79 \cdot 1(18 \cdot 8)$ & $77(22 \cdot 5)$ & $99(41)$ \\
FRC & $91 \cdot 9(20 \cdot 4)$ & $92 \cdot 8(18 \cdot 5)$ & $109(39 \cdot 5)$ \\
TLC & $100 \cdot 5(13 \cdot 6)$ & $91 \cdot 8(14 \cdot 7)$ & $105(21)$ \\
TLCO & $116 \cdot 5(30 \cdot 4)$ & $105 \cdot 2(30 \cdot 7)$ & $78 \cdot 8(40)^{\star}$ \\
$\mathrm{TLCO}_{\text {ss }}$ & $91(20 \cdot 9)$ & $96 \cdot 7(28 \cdot 6)$ & $59 \cdot 3(27)^{\star} \dagger$ \\
$\mathrm{PaCO}_{2}(\mathrm{~mm} \mathrm{Hg})$ & $39 \cdot 5(3 \cdot 2)$ & $38 \cdot 9(4 \cdot 3)$ & $39 \cdot 6(2 \cdot 8)$ \\
$\mathrm{PaO}_{2}(\mathrm{~mm} \mathrm{Hg})$ & $95 \cdot 1(12 \cdot 7)$ & $98(14 \cdot 6)$ & $92 \cdot 2(14 \cdot 5)$ \\
\hline
\end{tabular}

${ }^{\star} \mathrm{P}<0.05 v$ category $0 ;+\mathrm{P}<0.05 v$ category 1 .
Table 1 shows the distribution of the subjects in the different CR categories. There was no significant difference in the mean age, smoking, or duration of work between the CR categories, although subjects with higher profusion of small opacities were mainly ex-smokers or non-smokers. Symptoms of chronic bronchitis (according to the CIBA Guest Symposium criteria) were reported by 17 out of 27 subjects (63\%), without any significant difference between the different categories.

There was a mild concordance between $\mathrm{CR}$ and HRCT categories of parenchymal opacities. Only 10 out of 27 subjects were classified in the same category for $C R$ and HRCT (fig 1), and the largest discrepancy was found in the categories 0 and 1. Eight subjects were classified in the category 0 by CR; only two of these eight were classified in the category 0 by HRCT. Similar findings existed for the subjects classified in the category 0 by HRCT. The concordance between CR and HRCT was not influenced by smoking or symptoms of chronic bronchitis; in particular chronic bronchitis was reported in a similar percentage of subjects with category 0 by HRCT or CR (five out of eight subjects with category 0 by $C R$, and in four out of seven subjects with category 0 by HRCT).

No relation was found between pulmonary function tests and profusion of parenchymal opacities as assessed by CR, except for a reduction of maximal expiratory flow at $50 \%$ of FVC $\left(\mathrm{MEF}_{50}\right)$ in the highest CR category (table 2). On the contrary, the profusion of opacities detected by HRCT was associated with an impairment of the indices of airway obstruction (FEV $\left., \mathrm{MEF}_{50}, \mathrm{MEF}_{75}\right)$ and diffusion capacity (table 3 ); in particular, subjects with the highest category by HRCT showed lower values of $F E V_{1}$, expiratory flows, and diffusion capacity than subjects with category 0 or 1 . Subjects with simple silicosis by HRCT (categories 1 and 2) and subjects with conglomerated silicosis (categories 3 and 4) showed lower $\mathrm{FEV}_{1}$ and expiratory flows compared with subjects without silicosis (category 0 ), but subjects with conglomerated silicosis had higher values of RV and FRC than subjects with simple silicosis (fig 2). Evaluation of cigarette smoking in these subjects identified a significant relation with impairment of FRC and total lung capacity, but not with other spirometric indices. Multiple logistic regression analyses between pulmonary function tests and HRCT categories showed that the relation was not explained by smoking or symptoms of chronic bronchitis.

The HRCT evaluation of emphysema showed a significant reduction of $\mathrm{TLCO}_{\mathrm{SB}}$ (percentage of the predicted value) in subjects with the highest grades of emphysema (113 $(28.8) \%$ in grade $0,103.3(39.9) \%$ in grade 1 , and $63.9(24.1) \%$ in grade $2, P<0.05)$. There was also a significant correlation between the grades of emphysema and the HRCT categories of profusion $(r=0 \cdot 75, \mathrm{P}<$ 


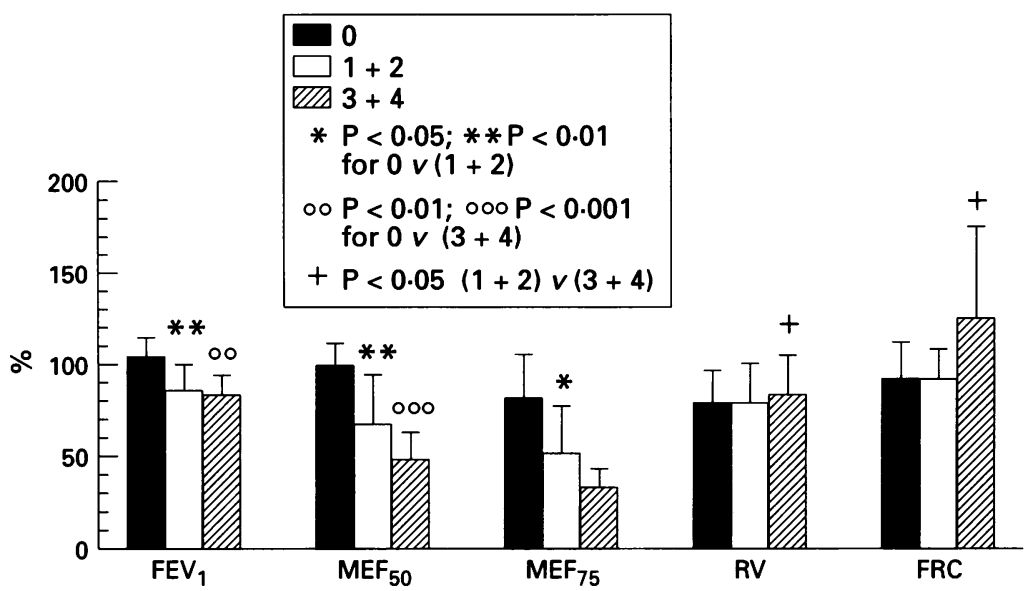

Figure 2 Mean values of pulmonary function tests in pooled HRCT categories of profusion of parenchymal opacities. or transbronchial or open biopsy. The few data published on this point concern mainly other interstitial lung diseases and only occasional cases of silicosis are reported ${ }^{24}$; furthermore, the relation between CT and morphology was only qualitative, and no comparison was attempted between the profusion of small opacities detected by HRCT and a morphological score of interstitial involvement. Other authors used the mineral content of bronchoalveolar lavage cells as a surrogate of the total lung burden of dust ${ }^{25}$; unfortunately, the technique of this analysis is not completely standardised, and a clear cut off point between normal and exposed subjects has not been reported. This technique, however, could identify the severity of the exposure to dust, but not the degree of the reaction of the lung parenchyma to silica dust.

Our data confirm the poor concordance between CR and HRCT in the early stages of the disease. In the absence of a gold standard for the diagnosis of silicosis, it is difficult to decide which technique is more accurate to detect early parenchymal involvement in silicosis. The better concordance between the readers for HRCT than for $C R$, similar to that reported $^{34}$ despite the absence of ILO type films for the CT images, and the presence of a significant correlation between HRCT categories and pulmonary function tests seem to indicate a better accuracy for HRCT than for CR.

The presence of a significant relation between the profusion of the parenchymal opacities evaluated by HRCT, and the pulmonary function tests has not been extensively reported in previous studies. Begin and coworkers showed that lung volumes, diffusion capacity, and expiratory flow rates were significantly reduced in the presence of complicated silicosis (coalescence or conglomeration of nodules on chest $x$ ray films or CT), but that there was a significant correlation between expiratory flow rates and profusion categories in subjects with simple silicosis. ${ }^{17}$ Some authors think that the functional abnormalities are almost completely explained by the presence of pulmonary emphysema, which

\footnotetext{
Figure 3 Percentage Figure 3 Percentage classes in a section of the upper lung field in different HRCT categories. Distribution of category 1 is significantly different from that of category $3+4$ $\left(\chi^{2}=6\right.$, by KolmogorovSmirnov test, $P<0.05$ ).
}

\begin{abstract}
Discussion
In recent publications there is some disagreement on the concordance between CR and CT in the evaluation of the profusion of lung parenchymal opacities in silicosis, especially in the initial stages. ${ }^{14-18}$ This discrepancy can be partially explained by the lack of a "gold standard" for the diagnosis of silicosis some methodological problems related to the technique of analysis, and to interpretation by the readers. A true gold standard for interpretation of $x$ ray films should be the morphology of the lung parenchyma obtained by necropsy
\end{abstract}

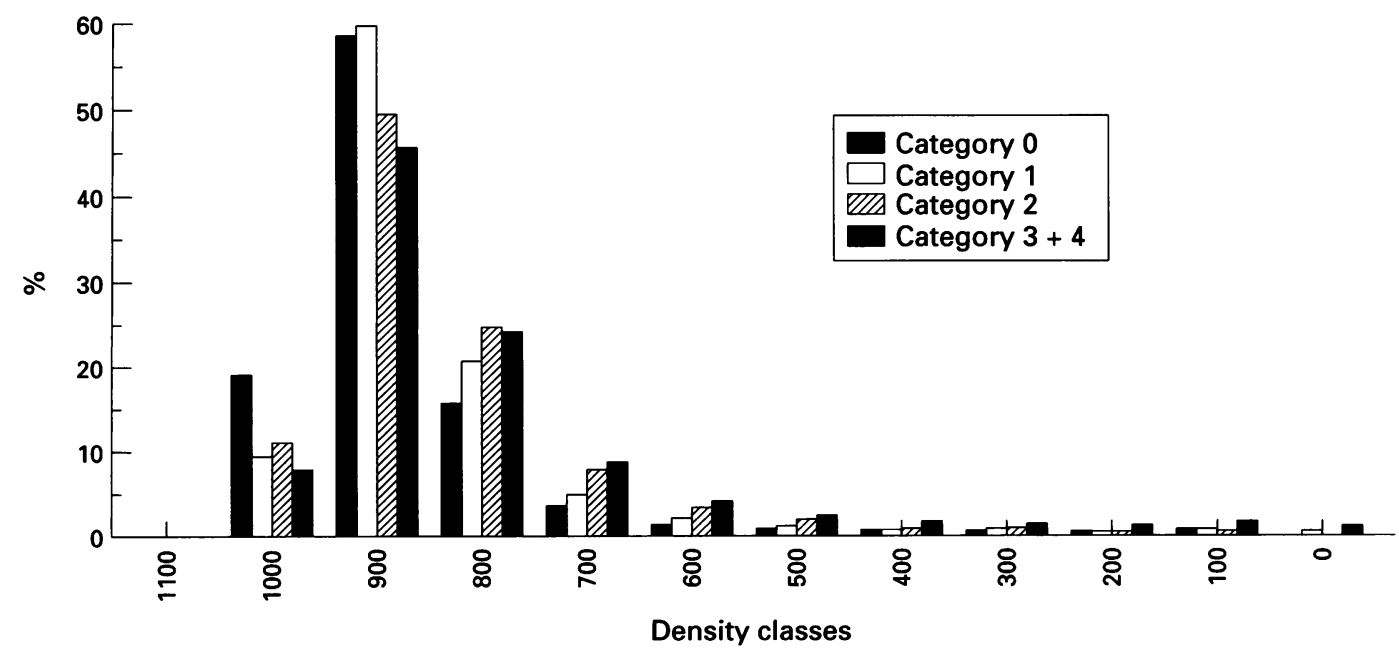


is mainly related to smoking. ${ }^{1618}$ Our study shows that the increasing severity in HRCT categories is associated with progressive deterioration in expiratory flows and diffusion capacity, whereas no relation could be found between CR categories and lung function. This finding has not been previously reported, and supports the argument that HRCT is more accurate in detection of functionally significant pulmonary changes. In particular, category 0 by HRCT selected subjects with normal lung function better than category 0 of $\mathrm{CR}$, whereas higher categories of HRCT are associated with higher abnormalities in lung volumes, expiratory flows, and diffusion capacity. A recent study shows that the degree of silicosis but not of emphysema at necropsy, was associated with a significant impairment of lung function in non-smoking gold miners, ${ }^{26}$ which suggests that silicosis can cause changes in pulmonary function without the contribution of smoking and emphysema. So as HRCT correlates better than CR with pulmonary function tests it can be argued that HRCT is more accurate than CR. Previous results had suggested that parenchymal nodules can also be found in symptomatic smokers without relevant airway obstruction. ${ }^{27}$ Thus we could argue that parenchymal opacities found by HRCT are mainly due to smoking and to the concomitant chronic bronchitis, which then explains the association with abnormal pulmonary function tests. The presence of similar percentages of smokers in our HRCT categories does not support this hypothesis, although the limitation of our sample does not allow a separate analysis of smokers and non-smokers. The results of the multiple logistic regression analysis between pulmonary function tests and HRCT categories, taking into account smoking and chronic bronchitis, showed that the relation found was not explained by these confounding factors.

As reported by other authors, ${ }^{17}$ HRCT detected the presence of the coalescence or conglomeration in silicosis better than $\mathrm{CR}$, and subjects with conglomerated silicosis by HRCT showed more impairment in pulmonary function than did subjects with simple silicosis. This fact was not found with $\mathrm{CR}$, which again supports HRCT as being more accurate in grading the severity of silicosis.

The extent of emphysema as detected by HRCT was in general mild in our subjects (all of them had a category $\leqslant 2$ ); nevertheless, a significant difference in diffusion capacity was found between the different categories of the extent of emphysema, which confirmed previous results. ${ }^{16} 18$ Recent data have confirmed a significant correlation between diffusion capacity and emphysema detected by CT in subjects with mild to moderate COPD. ${ }^{28} \mathrm{~A}$ significant correlation was found in our subjects between the severity of emphysema and the severity of nodular parenchymal opacities, which suggests that emphysema could be related to the silicosis and not only to associated smoking or chronic bronchitis. With the present data we cannot definitively assess the relative importance of silica exposure and smoking in the development of emphysema; more extensive evaluation on a larger number of subjects could allow a separate analysis of these features.

Finally, the study of the distribution of the classes of different density of the lung parenchyma detected by HRCT showed significant distinctions only between the extreme categories of HRCT. In asbestosis other authors showed a very high sensitivity of this technique of objective assessment of HRCT to select subjects with early asbestosis from normal subjects. ${ }^{29}$ The lower sensitivity of this technique in our experience could be explained by the simultaneous presence of emphysema and nodular parenchymal opacities in the different categories of HRCT, with conflicting influences on the distribution of the lung density classes. Although the weak significant difference among the different HRCT categories supports the validity of our HRCT classification, this technique seems not to have relevant usefulness in the clinical evaluation of silicosis.

In conclusion, our study suggests that HRCT is more reproducible and accurate than CR in the assessment of the severity of pulmonary involvement in silicosis, and in the selection of subjects with different pulmonary functional impairment, although there is no evidence that the sensitivity of HRCT is better than CR in the early detection of silicosis.

1 Graham WG. Silicosis. Clin Chest Med 1992;13: 253-67.

2 International Labour Office. Guidelines for the use of ILO international classification of radiographs of pneumoconiosis; revised ed. Geneva: ILO, 1980. (Occupational Safety and Health Series No 22.)

3 Muir DCF, Bernholz CD, Morgan WKC, Roos JO, Chan J Maehle W, et al. Classification of chest radiography fo pneumoconiosis: a comparison of two methods of reading. $B r \mathcal{F}$ Ind Med 1992;49:869-71.

4 Musch DC, Landis JR, Higging ITT, Gilson JC, Jonese RN. An application of Kappa-type analysis to interobserver variation in classifying chest radiographs for pneumoconiosis. Stat Med 1984;3:73-93.

5 Nemery B, Veriter C, Brasseur L, Frans A. Impairment of ventilatory function and pulmonary gas exchange in non smoking coal miners. Lancet 1987;ii:1427-30.

$6 \mathrm{Ng} \mathrm{TP,} \mathrm{Tsin} \mathrm{TW,} \mathrm{O'Kelly} \mathrm{FJ,} \mathrm{Chan} \mathrm{SL.} \mathrm{A} \mathrm{survey} \mathrm{of}$ the respiratory health of silica exposed gemstone workers in Hong Kong. Am Rev Respir Dis 1987;135 1249-54.

7 Rom WN, Bitterman PB, Rennard SI, Cantin A, Crystal $R$. Characterization of the lower respiratory tract inflammation of non smoking individuals with interstitial lung disease associated with chronic inhalation of inorganic dusts. Am Rev Respir Dis 1987;136:1429-34.

8 Bisson G, Lamoreux G, Begin R. Quantitative gallium-67 lung scan to assess the inflammatory activity in the pneumoconiosis. Semin Nuc Med 1987;17:72-80.

9 Mc Loud T. Occupational lung disease. Radiol Clin North Am 1991;29:931-41.

10 Remy-Jardin M, Remy J, Farre I, Marquette $\mathrm{CH}$ Computed tomographic evaluation of silicosis and coal Computed tomographic evaluation of silicosis and coal worker's pne 30 :1155-76.

11 Mathieson JR, Mayo JR, Staples CA, Muller NL. Chronic diffuse infiltrative lung disease: comparison of diagnostic accuracy of CT and chest radiography. Radiology 1989;171:111-6

12 Muller NL, Miller NN. State of the art: computed tomography of chronic diffuse infiltrative lung disease. $A m$ Rev Respir Dis 1990;142:1440-8.

13 Remy-Jardin M, Remy J, Deffontaines C, Duhamel A 
Assessment of diffuse infiltrative lung disease: comparison of conventional CT and high-resolution CT. Radiology 1991;181:157-62.

14 Begin R, Ostiguy G, Fillion R, Colman N. Computed tomography scan in the early detection of silicosis. $\mathrm{Am}$ Rev Respir Dis 1991;144:697-705.

15 Begin R, Bergeron D, Samson L, Boctor M, Cantin A. CT assessment of silicosis in exposed workers. $A \mathcal{F R} A m^{f}$ Roentgenol 1987;148:509-14.

16 Kinsella M, Muller NL, Vedal S, Staples C, Abboud RT, Chan-Yeung $M$. Emphysema in silicosis. A comparison of smokers with non-smokers using pulmonary function testing and computed tomography. Am Rev Respir Dis 1990;141:1497-1500.

17 Begin R, Ostiguy G, Cantin A, Bergeron D. Lung function in silica-exposed workers: a relationship to disease severity assessed by CT scan. Chest 1988;94:539-45.

18 Bergin CJ, Muller NL, Vedal S, Chan-Yeung M. CT in silicosis: correlation with plain films and pulmonary function tests. AfR Am $\mathcal{f}$ Roentgenol 1986;146: function

19 Paoletti P, Pistelli G, Fazzi P, Viegi G, Di Pede F, Giuliano G, et al. Reference values for vital capacity and flow-volume curves from a general population study. Bulletin of European Physiopathology and Respiration 1986;22:451-9. 20 Quanjer PHII, ed. Standardized lung function testing. 1983;19(suppl 5):95.

21 Cotes JE. Lung function at different stages of life, including reference values. In: Cotes JE, ed. Lung function, $3 r d$ ed. Oxford: Blackwell Scientific Publications, 1975.

22 Bates DV, Macklem PT, Christie RV. Respiratory function in diseases. Philadelphia: Saunders, 1971.

23 Armitage P. Statistica medica. Milano: Feltrinelli, 1981.

24 Mathieson JR, Mayo JR, Staples CA, Muller NL. Chronic diffuse infiltrative lung disease: comparison of diagnostic accuracy of CT and chest radiography. Radiology accuracy of CT

25 Lusuardi M, Capelli A, Donner CF, Capelli O, Velluti G. Semi-quantitative $x$ ray microanalysis of bronchoalveolar lavage samples from silica-exposed and non-exposed subjects. Eur Respir F 1992;5:798-803.

26 Hnizdo E, Sluis-Cremer GK, Baskind E, Murray J. Emphysema and airway obstruction in non-smoking South African gold miners with long exposure to silica dust. Occup Environ Med 1994;51:557-63.

27 Remy-Jardin M, Remy J, Boulanguer C, Sobaszek A, Edue $J$ Furon D. Morphologic effect of cigarette smoking on Jirways and pulmonary parenchyma in healthy adult airways and pulmonary parenchyma in healthy adult volunteers: CT evaluation and correlation with pul-
monary function tests. Radiology 1993;186:107-17.

monary function tests. Radiology 1993;186:107-17.
28 Gelb AF, Schlin M, Kuei J, Tashkin DP, Muller NL, Hogg JC, et al. Limited contribution of emphysema in advanced chronic pulmonary disease. Am Rev Respir Dis 1993;147:1157-61.

29 Eterovic D, Dujic Z, Tocilj J, Capkun V. High resolution pulmonary computed tomography scans quantified by analysis of density distribution: application to asbestosis. $\mathrm{Br}$ F Ind Med 1993;50:514-9. 